\title{
Friction utilization for tyre-road friction estimation on snow: an experimental study
}

\author{
A. Albinsson, F. Bruzelius, B. Jacobson \\ Department of Applied Mechanics, Chalmers University of Technology, Gothenburg, Sweden
}

\begin{abstract}
Friction estimation using effect-based approaches are challenging during normal driving due to the large tyre excitation needed for an accurate estimate. The required excitation level varies for different tyres, road surfaces, road conditions and tyre models used in the estimator. Previous research has investigated the required friction utilization on different surfaces but due to the small sample sizes it is hard to draw any general conclusions. This paper investigates the tyre excitation required to estimate the tyre-road friction coefficient with a generic estimator for 76 different tyres on snow for five different tyre models and for different levels of measurement noise.
\end{abstract}

\section{INTRODUCTION}

The maximum tyre-road friction limits the horizontal forces that the tyres can generate. Knowledge about the tyre-road friction coefficient is thus useful for active safety and driver assistance systems where the appropriate intervention is dependent on the maximum available road grip. Vehicles with increasing levels of autonomous functions, such as autonomous emergency braking or, in the future, fully autonomous vehicle, can use this information to adapt the intervention thresholds or vehicle velocity based on the prevailing road conditions. The information can also be shared with other drivers or vehicles to warn them of an approaching area with low friction.

The tyre-road friction coefficient is difficult to estimate during normal driving, mainly due to the limited information about the maximum achievable tyre forces in the linear tyre region, see (Lex, 2015) and (Ray, 1997). Previous research have attempted to correlate the slip stiffness to the maximum road friction coefficient to remove the need for large tyre excitation (Gustafsson, 1997). However, the relation between the friction coefficient and the slip stiffness relies on empirical data. A-priori information about the tyres that are currently fitted to the vehicle is thus required. Another approach was evaluated experimentally in (Albinsson et al., 2015). Opposite wheel torques were added to the front and the rear axle to achieve large tyre excitation during normal driving, see also (Chen and Wang, 2011). This method, active tyre force excitation, makes it possible to achieve large tyre forces during normal driving and thus to estimate the friction coefficient.

The required utilization, i.e. here defined as the ratio of the required and maximum normalized longitudinal force, to estimate the friction coefficient within an accuracy of $\pm 15 \%$ for different tyres and road surfaces is investigated in (Svendenius, 2007). However, only a few different tyres were evaluated using the brush model and it is therefore difficult to draw any general conclusions.

This study investigates how well the tyre-road friction coefficient can be estimated by fitting a non-linear tyre model to measurements on a snow surface for 76 different tyres. The estimation error that can be expected without any a-priori information about the tyre can thus be evaluated with a large sample size. Furthermore, the estimation error for different tyre models are evaluated and compared, thus indicating which tyre models that are more suitable for friction estimation on snow. Noise was added to the measurements signals to investigate how different fitting error cost functions influence the estimation accuracy.

\section{METHOD}

The measurements were performed for another study with the BV12 measurement vehicle from VTI (The Swedish National Road and Transport Research Institute), (Hjort and Eriksson, 2015). Four to six slip ratio sweeps up to a fully locked wheel was done in each test run. The data was filtered and the offset at 
zero slip was removed so that the tyre force is zero at zero longitudinal slip. The data was used as the reference tyre characteristics when evaluating the different tyre models. These tyre models assumes steady-state conditions and the slip ratio gradient should thus be kept small.

During the measurements, the maximum slip ratio gradient was around $0.2\left[\left(m s^{-1}\right) /\left(m s^{-1}\right) 1 / s\right]$ at small tyre forces. With a first order relaxation model, Equation 1, the difference between the nominal $\sigma_{(x, n o m)}$ and the actual slip ratio $\sigma_{x}$ can be calculated as,

$$
\begin{aligned}
& \dot{\sigma}_{x}=\left(\sigma_{x, n o m}-\sigma_{x}\right) \frac{v_{x}}{r_{l x}} \\
& \left(\sigma_{x, n o m}-\sigma_{x}\right)=\dot{\sigma}_{x} \frac{r_{l x}}{v_{x}}
\end{aligned}
$$

where $r_{l x}$ is the relaxation length and $v_{x}$ is the longitudinal velocity of the vehicle. Assuming that the relaxation length is 0.1 and with the longitudinal velocity of $8.33 \mathrm{~m} / \mathrm{s}$ the difference between the nominal and the actual slip ratio is $0.0024\left[\left(\mathrm{~ms}^{-1}\right) /\right.$ $\left.\left(m s^{-1}\right)\right]$. At a slip level of $0.05\left[\left(\mathrm{~ms}^{-1}\right) /\right.$ $\left.\left(m s^{-1}\right)\right]$ this corresponds to a $5 \%$ error.

The tyre relaxation will hence have an influence on the tyre characteristics and so will other measurement errors. However, the characteristics of specific road-tyre combinations are not interesting in themselves for the purpose of this study but rather the variation in the tyre-road characteristics represented by these different tyres.

The tested tyres were 9 new and 17 used studded tyres, 9 new and 16 used Nordic non-studded winter tyres, and 9 new and 16 used European non-studded winter tyres.

\subsection{Tyre Models and Tyre Parameter Fitting}

Four different tyre models are evaluated, the brush model with parabolic pressure distribution, the magic tyre formula with 4 parameters (Bakker et al., 1987), Burckhardt tyre model and Dugoff tyre model (Dugoff et al., 1969), see Equation 3-6. These tyre models are commonly used for online friction estimation, where the number of parameters is a concern due to the limited number of measurement points and since the estimation is normally done recursively.

$$
\begin{gathered}
f\left(\sigma_{x}\right) \\
=\left\{\begin{array}{c}
-C \sigma_{x}+\frac{C^{2} \sigma_{x} \mid \sigma_{x \mid}}{3 \mu_{\max }}-\frac{C^{3} \sigma_{x}^{3}}{27 \mu_{\max }^{2}} \text { for }\left|\sigma_{x}\right| \leq \frac{3 \mu_{\max }}{C} \\
-\operatorname{sign}\left(\sigma_{x}\right) \mu F_{z} \text { else }
\end{array}\right. \\
f\left(\sigma_{x}\right)=D \sin \left(C _ { m } \operatorname { t a n } ^ { - 1 } \left(B \sigma_{x}\right.\right. \\
\left.\left.-E\left(B \sigma_{x}-\tan ^{-1}\left(B \sigma_{x}\right)\right)\right)\right)
\end{gathered}
$$

$f\left(\sigma_{x}\right)=c_{1}\left(1-e^{c_{2} \sigma_{x}}-c_{3} \sigma_{x}\right)$

$$
\begin{aligned}
& f\left(\sigma_{x}\right)=\left\{\begin{array}{c}
C \sigma_{x}(2-\lambda) \lambda \text { for }|\lambda|<1 \\
C \sigma_{x} \text { else }
\end{array}\right. \\
& \lambda=\frac{\mu_{\text {max }}}{2\left|C \sigma_{x}\right|}
\end{aligned}
$$

The tyre model parameters are fitted to the measurements to minimize two different cost functions,

$$
J_{f}=\sum_{i=1}^{N}\left(f_{m}[i]-f_{d}[i]\right)^{2}
$$

$$
\begin{gathered}
\left.J_{s}=\sum_{i=1}^{N}\left(f_{m}[i]-f_{d}[i]\right)^{2}\right)+\left(\frac { \partial f } { \partial \sigma _ { d } } | _ { \sigma _ { d } [ i ] } \left(\sigma_{m}[i]\right.\right. \\
\left.\left.-\sigma_{d}[i]\right)\right)^{2}
\end{gathered}
$$

$f_{m}$ is the normalized force from the tyre model at the measured slip ratio, $f_{d}$ is the measured normalized force, $\sigma_{d}$ is the measured slip ratio and $\sigma_{m}$ is the slip ratio at the measured normalized force obtained from the inverse tyre model. The cost function $J_{f}$ in Equation 7 does not penalize the slip ratio error and should thus reasonably make the tyre parameter estimates more sensitive to measurement noise in the slip measurements. Equation 8 is inspired by the total least square approach but has been simplified so that the terms in the cost function is available directly from the tyre model. Figure 1 illustrates the different components in the cost functions, $J_{f}$ penalizes $\Delta f, J_{S}$ penalizes $\Delta f$ and $\Delta \sigma$.

By using the cost function in Equation 8 rather than the total least square method the evaluation of the cost function is made computationally more efficient. A total-least square approach use the perpendicular distance between the measurement point and the model. However, the expressions for finding the corresponding model point $\sigma_{m}, f_{m}$ are nonlinear and not easily solved analytically which results in the high computational cost. The cost function $J_{S}$ is hence a compromise to get an expression that penalizes both the slip and force error that is easily obtained from the tyre models and which is easy implement online.

The parameters of the tyre models were found using the gradient based optimization function fmincon with the interior-point algorithm in Matlab. The parameters of the tyre models were constrained to obtain reasonable tyre characteristics, Table 1 . The gradient based optimization approach can in some circumstances converge to a local minimum. The cost function should hence preferably have one distinct minimum and not be too flat.

Table 1, Tyre parameter constraints

\begin{tabular}{|c|l|l|l|}
\hline$C$ & {$[2,100]$} & $\mathrm{B}$ & {$[1,100]$} \\
\hline$\mu_{\max }$ & {$[0.05,1.5]$} & $c_{1}$ & {$[0.05,1.5]$} \\
\hline$D$ & {$[0.05,1.5]$} & $c_{2}$ & {$[0,50]$} \\
\hline$E$ & {$[-1.5,1]$} & $c_{3}$ & {$[-0.01,0.01]$} \\
\hline$C_{m}$ & {$[1,1],[1,1.6]$} & \multicolumn{2}{|l|}{} \\
\hline
\end{tabular}




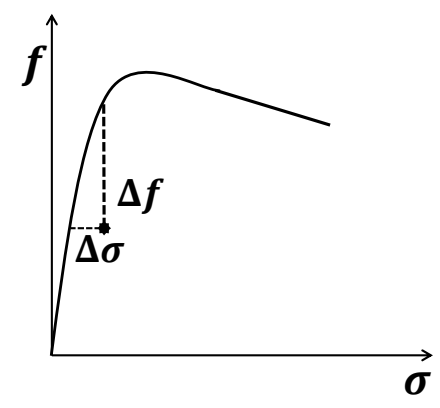

Figure 1. Cost function illustration

\subsection{Evaluation Procedure}

By using the reference tyre data from the measurements as a lookup table, an arbitrary excitation can be used to evaluate the different tyre models. However, it has to be ensured that the time derivative of the slip ratio is kept small enough to keep the tyre relaxation negligible. The excitation levels are described by the friction utilization $\mu_{\mathrm{util}}$, defined here as the ratio between the normalized force $\mu$ and the maximum friction coefficient $\mu_{\max }$.

The normalized estimated friction coefficient $\hat{\mu}_{n o r m}$ and the normalized friction estimation error $\tilde{\mu}_{\text {norm }}$ are defined as,

$$
\begin{aligned}
\hat{\mu}_{\text {norm }} & =\frac{\hat{\mu}_{\text {max }}}{\mu_{\text {max }}} \\
\tilde{\mu}_{\text {norm }} & =\frac{\hat{\mu}_{\text {max }}-\mu_{\max }}{\mu_{\max }}
\end{aligned}
$$

These measures can be used to compare the results from different tyres since they are normalized with the maximum friction coefficient. The evaluations are done for different levels of white Gaussian noise added to the slip ratio and force signal. The noisy environment highlights the differences between the two different cost functions. The added noise signals are uncorrelated but has the same variance $\sigma^{2}$. The lower noise level, $\sigma^{2}=0.003^{2}$, approximately corresponds to the noise levels found for the force and slip estimates during active tyre force excitation but with a lower variance for the force estimation (Albinsson et al., 2015). Other disturbances, due to for instance suspension compliance or uneven road surfaces, are not represented by the added noise. These disturbances will influence the measurements in reality but experience a behaviour that is hard to model and analyse.

\section{RESULTS}

The mean of the tyre-road friction coefficient estimate, normalized with the measured maximum friction coefficient, for the different tyre models as a function of the utilized friction are shown in Figure 2.
AVEC'16 The standard deviation of the estimate is shown with an error bar.

The level of utilization required to obtain a friction estimation error of below $10 \%$ and $20 \%$ for two different noise level is presented in Figure 3-6. The noise level is the same on both the force and the slip ratio signal. Note that the limit of $10 \%$ and $20 \%$ is strict. The Dugoff model with noise std $=0.005$ and using the force cost function $J_{f}$ is close to the $10 \%$ error limit already from $75 \%$ utilization, see Figure 3.

The value of the cost functions for different tyre parameters has been plotted for one of the tyres with noise for the Dugoff model, see Figure 8. Different noise levels are used on the force and the slip ratio signals to more closely represent the noise found in previous work, see (Albinsson et al., 2015). Note that the cost function values has been limited to have the same scale in the different figures. The cost function $\mathrm{J}_{\mathrm{f}}$, which only penalizes the force error, does not have as distinct boundary at the lower friction values as the cost function $J_{S}$. The cost function $J_{S}$ thus penalizes low friction estimates more than the cost function $\mathrm{J}_{\mathrm{f}}$. This means that the friction is more likely to be underestimated at low friction utilization when the cost function $\mathrm{J}_{\mathrm{f}}$ is used compared to using the cost function $\mathrm{J}_{\mathrm{s}}$.

The reason for this can be understood by looking at Figure 1. If noise is present on both the slip signals and the force signal and only penalizes deviations in the force, measurement points as indicated in Figure 2 must be fitted by changing the friction coefficient. However, if the slip error is penalized as well the influence of these measurement points on the friction estimation is smaller. The tendency to underestimate the friction coefficient when only penalizing the force estimation can be seen in Figure 9 where the distribution of the normalized friction estimation error for low utilization is plotted.

\section{DISCUSSION}

The brush model with parabolic pressure distribution does not perform as well as the other tyre models. The mean friction estimate is lower than the current utilization even at high utilization levels. This is due to the poor fit that the brush model has for tyre characteristics on snow, see Figure 7. The difference in shape between the data and the tyre model makes it difficult to find tyre parameters that provides a good fit.

The Dugoff model fits the snow measurements better than the brush model. The models are based on the same basic assumptions but with different vertical pressure distribution in the contact patch. Both these models have only two parameters and have therefore difficulty to fit the data when the curvature of the nonlinear tyre region does not match the one described by the model. 

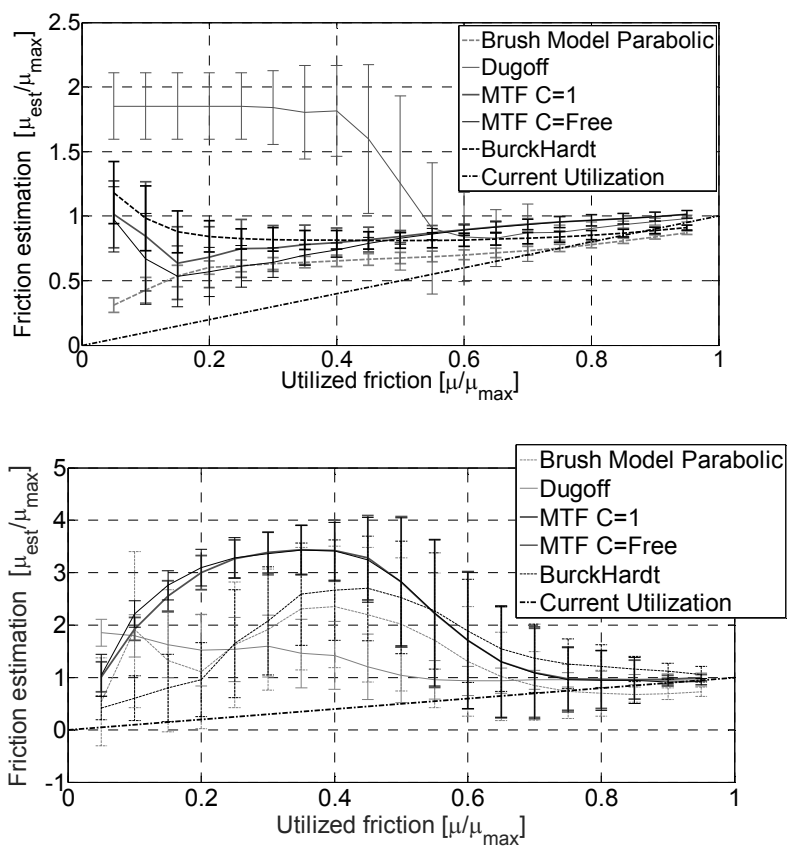
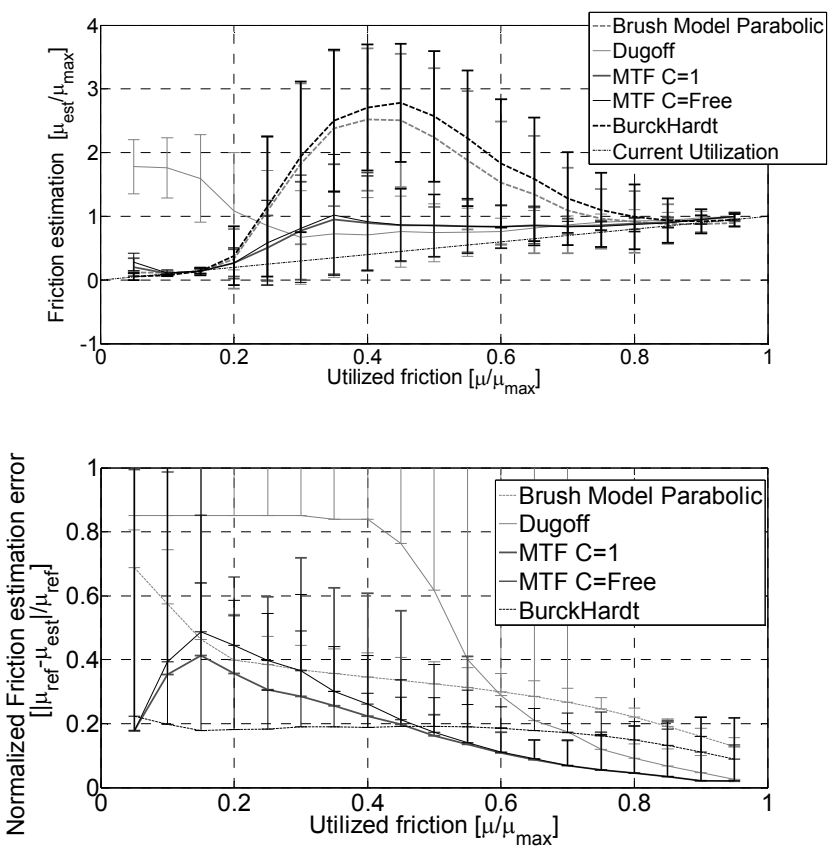

Figure 2. Mean normalized friction estimation of the measurements for the 76 tyres as a function of utilized friction with standard deviation shown as error bars. The current friction utilization is plotted as a dotted black line. Top left) Noise free input signals and minimizing force error. Top right) White Gaussian measurement noise $\left(\sigma^{2}=0.005^{2}\right)$ added to the slip and force and minimizing force error. Bottom left) noise added to the slip and force and minimizing force \& slip error (Equation 2). Bottom right) Mean and maximum friction estimation error, noise-free environment, force error minimization

Required excitation in \% for $10 \%$ mean error, Force

Error minimization $\mathrm{J}_{\mathrm{f}}$

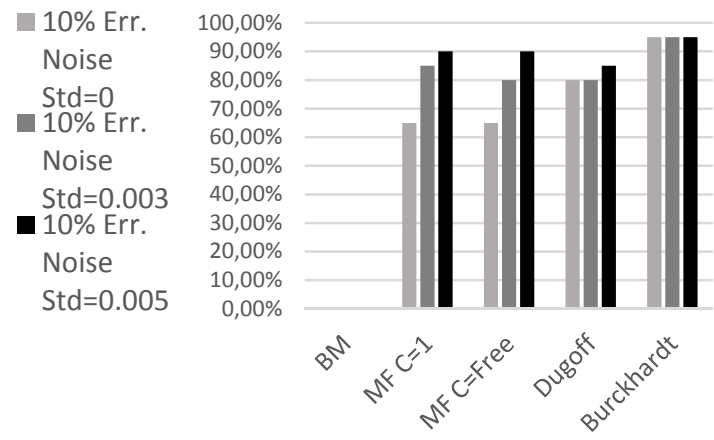

Figure 3. Required excitation in $\%$ for $10 \%$ mean error, Force Error minimization $J_{f}$

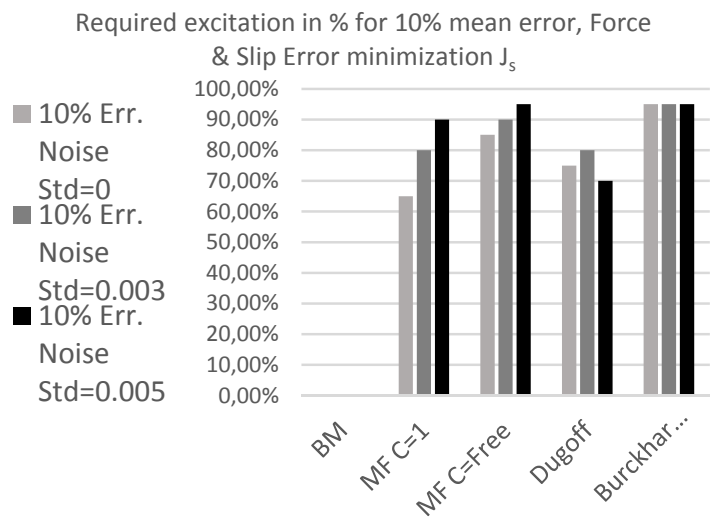

Figure 4. Required excitation in $\%$ for $10 \%$ mean error, Force and Slip Error minimization $J_{s}$

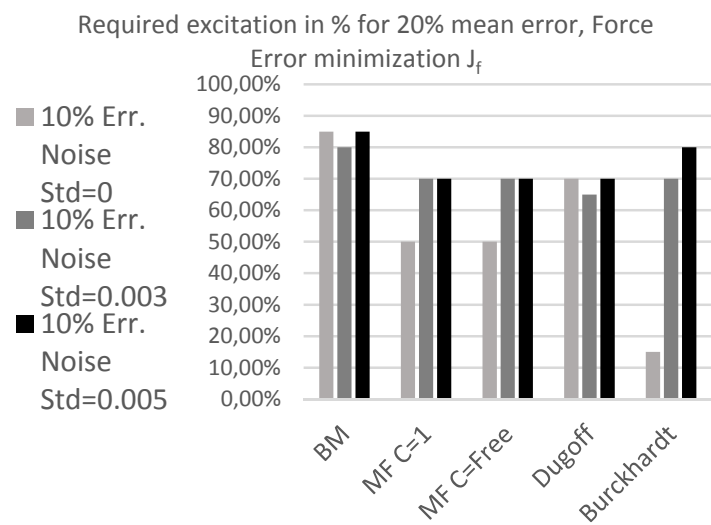

Figure 5. Required excitation in $\%$ for $20 \%$ mean error, Force Error minimization $J_{f}$

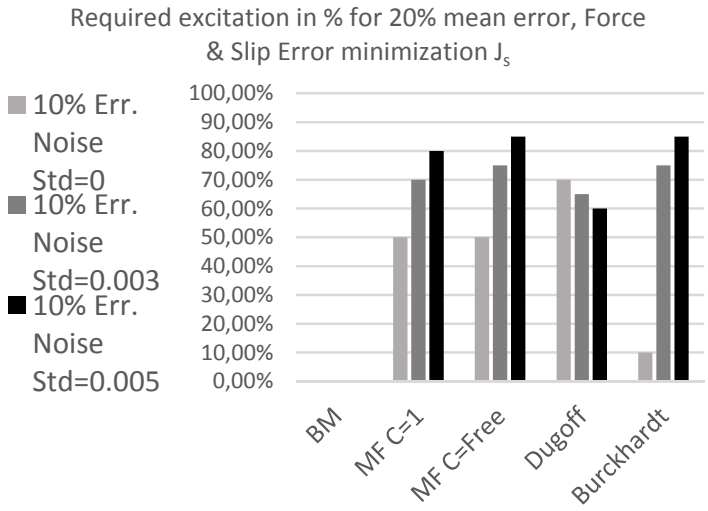

Figure 6. Required excitation in $\%$ for $20 \%$ mean error, Force and Slip Error minimization $J_{S}$ 
The Magic formula and the Burckhardt model have more than two parameters and can fit the snow measurements better. As seen from Figure 3-6, the magic tyre formula has the lowest required utilization for a $10 \%$ error in the friction estimate without any measurement noise.

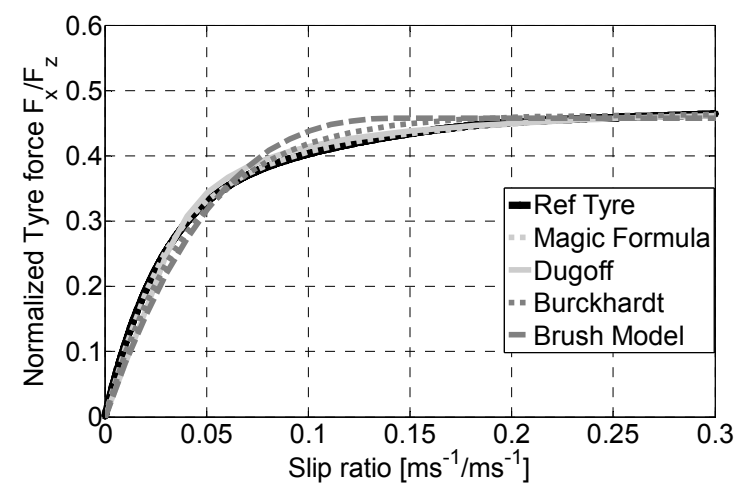

Figure 7. Example of brush model modelling error.

When adding noise, the performance of the Dugoff model does not decrease much in terms of required utilization. This makes it one of the models which require the lowest excitation levels in a noisy environment. With measurement noise, the Dugoff tyre model has lower required utilization than the magic

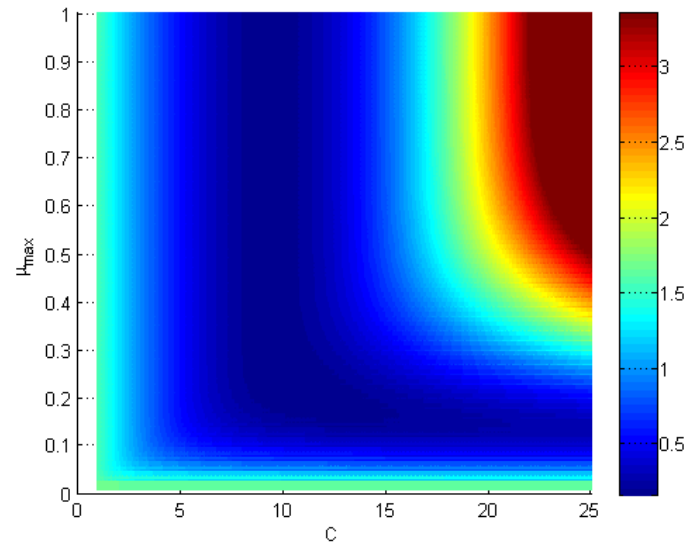

formula. Both the Magic tyre formula together with the Dugoff tyre model shows good promise to separate between low and high friction at low excitation and to achieve accurate friction estimation at larger excitation. The tyre model of choice should thus depend on the expected noise levels. However, it is important to note that these results are only valid for one road surface. On other road surfaces the tyre models may not perform as well. Similar studies should thus be performed on other road surfaces to investigate the change in friction estimation error for the different road surfaces. The Burckhardt model maintains the same performance in terms of required utilization (for ten percent error) when adding noise, however the Burckhardt model seems to require a larger excitation level compared to the magic formula and the Dugoff model.

\subsection{Cost function and measurement noise}

When noise is present on both the force and the wheel speed signal, only minimizing the force error can provide poor estimates since the friction coefficient estimate tends to follow the current utilization at low tyre utilizations.

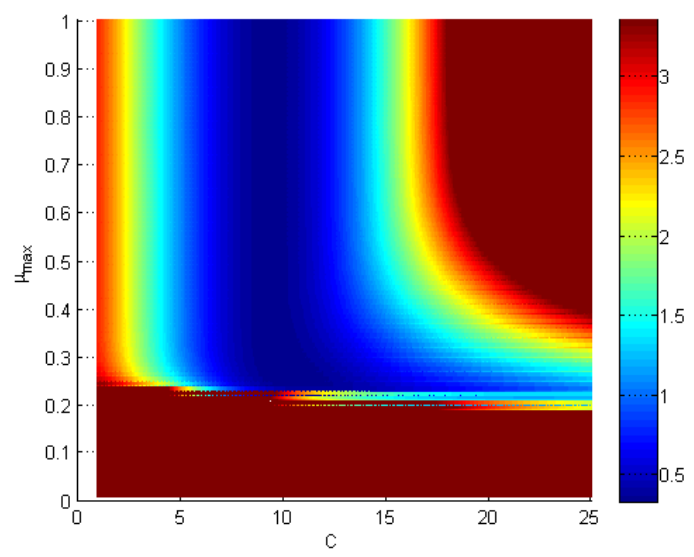

Figure 8. Cost function value for Dugoff tyre model for one of the tyres, with added noise on the force signal $\sigma_{f}^{2}=0.03^{2}$ and the slip signal $\sigma_{\sigma_{x}}^{2}=0.003^{2}$. Right plot for $J_{s}$, left plot for $J_{f}$. All plots at $30 \%$ friction utilization with $\mu_{\text {ref }}=0.54$.

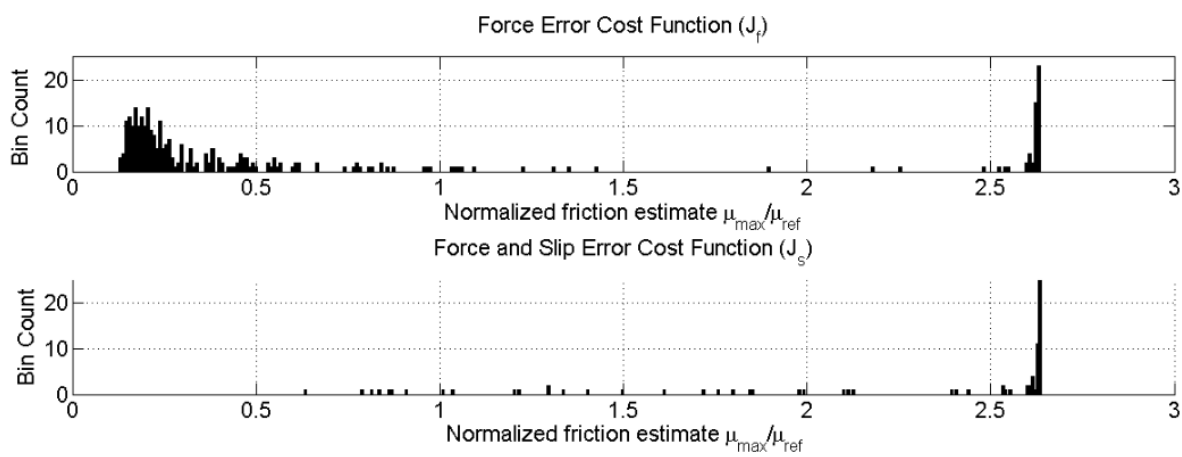

Figure 9. Normalized Friction estimation distribution $\mu_{\max } / \mu_{\text {ref }}$ for the brush model at $20 \%$ friction utilization and for 300 noise realizations. Force noise variance $\sigma_{f}^{2}=0.03^{2}$ and slip noise variance $\sigma_{\sigma_{x}}^{2}=0.003^{2}$. 
The additional slip term in the alternative cost function, Equation 2, creates a more distinguished separation between low and high friction at low tyre utilizations, see Figure 2. It penalizes low friction estimates more compared to only minimizing the force error, see Fig 10. Although the actual friction coefficient is unknown, the separation makes it possible to distinguish between high- and low-friction surfaces at low excitation levels. The Dugoff model is not as sensitive to measurement noise as the other models at low excitation. This is likely due to the fact that the Dugoff model is exactly linear at low tyre excitations. The cost function should thus be chosen depending on the noise level and the chosen tyre model.

\subsection{Required Tyre Excitation}

The required tyre excitation to estimate the friction coefficient with an average error of less than $10 \%$ and $20 \%$ is presented in Figure 3-6. As seen from the figures, the lowest required excitation can be found for the magic tyre formula (both with locked and estimated $C_{m}$ parameter) that has a required excitation of $65 \%$ (for $10 \%$ error) in a noise free environment. As expected the required excitation increases for increasing noise levels and with a white Gaussian noise with a standard deviation of 0.005 the required excitation has increased to $90 \%$ for the magic formula. This trend is similar for both cost functions.

Naturally, noise and disturbances cannot be avoided in the real-world application where compliances in the suspension, uneven road surfaces, varying pitch angle all affect the measurement signals. These disturbance are most likely not zero mean white Gaussian noise as used in this study and may further increase the need for large excitations. Nevertheless, the results illustrates the importance of actively working towards minimizing any noise and disturbances.

It should also be noted that this is the excitation required to reach a mean error of below $10 \%$. The error distribution is not shown here. However, it was found that at the excitation level required for $10 \%$ mean error in a noise free environment the maximum error was within $15 \%$ for all tyre models.

In this study, 200 samples were used for friction estimation. This corresponds to $2 \mathrm{~s}$ in a system with $100 \mathrm{~Hz}$ sampling time. With the vehicle used in previous studies, see (Albinsson et al., 2015), which had a maximum torque gradient of $1200 \mathrm{Nm} / \mathrm{s}$ the maximum front axle torque that can be reached is 2400 $\mathrm{Nm}$ or approximately $7500 \mathrm{~N}$. For the vehicle in question it is enough to reach a normalized force of $0.92[N / N]$ and thus enough to achieve $100 \%$ friction utilization on snow. Naturally the required torque may not be reached at higher velocities for vehicles with less powerful engines.

\section{CONCLUSION AND FUTURE WORK}

The brush model is not suitable for friction estimation on snow. The friction estimation error never goes below $10 \%$ and it has large modelling errors, see Figure 3-6 and Figure 7. The Dugoff model and the Magic formula on the other hand performs better with a lower required excitation. The Magic Formula requires lower excitation levels in a noise free environment. However, when adding noise to the slip and force signal the difference between the Dugoff Model and the Magic Formula in terms of required excitation is smaller. With noise added to the slip and force signals, the required excitation generally increase. In order to increase the performance of the estimator the noise and disturbance should hence be reduced as much as possible.

Similar measurements have been performed on wet asphalt and a similar study should be performed for these measurements as well. It is expected that the relative performance of some tyre models will change when the surface is changed from snow to wet asphalt due to modelling errors which favours one of the surfaces.

\section{REFERENCES}

Albinsson, A., Bruzelius, F., Gustafsson, T., Jonasson, M. \& Jacobson, B. Identification of tyre characteristics using active force excitation. 24th International Symposium on Dynamics of Vehicles on Roads and Tracks, 2015 Graz, Austria.

Bakker, E., Nyborg, L. \& Pacejka, H. B. 1987. Tyre modelling for use in vehicle dynamics studies. SAE Technical Paper.

Chen, Y. \& Wang, J. 2011. Adaptive vehicle speed control with input injections for longitudinal motion independent road frictional condition estimation. Vehicular Technology, IEEE Transactions on, 60, 839-848.

Dugoff, H., Fancher, P. S. \& Segel, L. 1969. Tire performance characteristics affecting vehicle response to steering and braking control inputs. Ann Arbor, Michigan: Highway Safety Research Institute.

Gustafsson, F. 1997. Slip-based tire-road friction estimation. Automatica, 33, 1087-1099.

Hjort, M. \& Eriksson, O. 2015. Test av is- och snögrepp för slitna vinterdäck. Jämförelse av olika kategorier av vinterdäck. www.vti.se Swedish National Road and Transport Research Institute (VTI).

Lex, C. 2015. Estimation of the Maximum Coefficient of Friction between Tire and Road Based on Vehicle State Measurements. $\mathrm{PhD}$ dissertation, Graz University of Technology.

Ray, L. R. 1997. Nonlinear tire force estimation and road friction identification: simulation and experiments. Automatica, 33, 1819-1833.

Svendenius, J. 2007. Tire modeling and friction estimation. Lund University. 